\title{
Instability of individual differences in the association between confidence judgments and memory performance
}

\author{
W. BURT THOMPSON and SUSAN E. MASON \\ Niagara University, Niagara Falls, New York
}

\begin{abstract}
There are large individual differences in the degree of association between the accuracy of memories and subjective confidence in those memories. Are these differences stable within the same test, and between alternate forms of a test? In Experiment 1, college students were tested on 3 recognition memory tasks, then retested 2 weeks later on alternate forms of the same tasks. The relationship between confidence judgments and recognition performance displayed low split-half stability and low alternate-forms stability. A second experiment with elderly adults replicated these findings. In a third experiment, college students recalled answers to general knowledge questions and rated confidence in the correctness of each answer. Individual differences in the association between confidence and recall performance were not stable across the odd- and even-numbered items on the test. These data indicate the need for the development of procedures that will produce stable estimates of individuals' metacognitive accuracy.
\end{abstract}

The present research investigated the association between the accuracy of memories and a person's confidence in those memories, a topic that has long been of interest in psychology (see, e.g., Seward, 1928; Strong, 1912, 1913; Trow, 1923). The association between confidence judgments and memory performance has been important in several areas of research, and currently is one aspect of metacognition being studied along with others such as feelingof-knowing judgments, judgments of learning, and calibration of comprehension. Also, in the eyewitness memory field, the usefulness of eyewitness confidence as a predictor of recall and recognition accuracy has been the subject of much research (e.g., Brigham, 1990; Deffenbacher, 1980; Leippe, 1980; Luus \& Wells, 1994; Perfect, Watson, \& Wagstaff, 1993; Wells \& Murray, 1984).

Individuals can vary greatly in metacognitive accuracy, that is, how accurately their metacognitive judgments predict performance on a criterion task. For example, Deffenbacher, Leu, and Brown (1981) reported that individual subjects' point-biserial correlations between confidence judgments and performance on a face recognition task ranged from -.05 to .60 . In the research presented in this article, Goodman-Kruskal gamma correlations between confidence and memory performance covered almost the entire possible range, from -1 to +1 . The magnitude of these individual differences is not confined to studies in which

Portions of these data were presented at the annual meeting of the American Psychological Association, Boston, August 1990. We thank R. Crowder, R. Fisher, A. Glenberg, C. MacLeod, T. Nelson, and R. Proctor for their helpful reviews of earlier versions of this article. We also thank J. Allen, R. Bonner, and D. DeMare for assisting with data collection. Correspondence should be addressed to W. B. Thompson, Department of Psychology, Niagara University, Niagara Falls, NY 14109 2208 (e-mail: wbt@niagara.edu). subjects make postrecall and postrecognition confidence judgments; correlations between feeling-of-knowing judgments (FOK) and recognition performance can vary greatly from person to person. Nelson (1988) reported a study in which FOK accuracy (indexed by the Goodman-Kruskal gamma coefficient) was measured for each individual in 3 groups of 42 college students. The 3 groups differed in the number of items used to compute each gamma. When gammas were based on 15,35, and 55 items, the standard deviations of individual gammas were $.27, .18$, and .14 , respectively. These data indicate that the range of individual gammas was greater than .50 (i.e., more than 2 standard deviations above and below the mean) even in the group for which there was the least variability.

In this article we focus on the instability of the individual differences that have been observed in metamemory accuracy, and more specifically, instability in the association between memory performance and confidence judgments. The issue here is whether the interindividual variability in the accuracy of metamemory judgments reflects consistent individual metamemory functioning or error variance. If individuals do exhibit stable differences in the accuracy of their metamemory judgments, an analysis of these differences would contribute to our understanding of metamemory. One significant result of a better understanding of individual differences in the association between confidence and memory performance might be the development of specific methods for improving that association. Such techniques could be applied in education, eyewitness testimony, cognitive neuropsychology, and other fields in which it is crucial for confidence to be a reliable index of memory performance.

Previous research has demonstrated that both confidence judgments and FOK judgments exhibit excellent testretest reliability for a given set of items. In other words, the 
metamemory judgment given to an item at the first test session is an excellent predictor of the metamemory judgment that will be given when the same item is presented at a second test session. Costermans, Lories, and Ansay (1992) asked subjects to answer 250 general knowledge questions. These 250 questions were presented twice in the same day, and at each test session subjects rated how confident they were in the accuracy of each answer they recalled. For questions they could not answer, the subjects made FOK ratings and took a true-false recognition test to measure the accuracy of their FOK ratings. For the questions that were answered twice, the test-retest reliability of the confidence judgments was .87 (assessed with the Goodman-Kruskal gamma coefficient); the reliability of FOK judgments given to questions that were not answered at either test session was also very high (.82). Nelson and his colleagues have also reported very high test-retest reliability for FOK judgments (e.g., Nelson, Leonesio, Landwehr, \& Narens, 1986).

It appears, then, that interindividual differences in metamemory judgments, such as FOK ratings and confidence judgments, are stable when the same test items are presented twice. But, are measures of metacognitive accuracy (i.e., the degree of association between metamemory judgments and memory performance) stable when assessed with different sets of items? This is an important question because in some situations, researchers may want to assess metacognitive accuracy at two different times and avoid the problem that arises when the same test items are used twicethe subjects may remember how they answered the first time. For example, a researcher may wish to conduct a within-subject experimental test of a technique designed to improve metacognitive accuracy, or to assess changes in metacognitive accuracy in a longitudinal developmental or neuropsychological study. In cases like these, it would be necessary for the researcher to use a measure of metacognitive accuracy for which individual differences are stable across different sets of items.

There are several standard methods for assessing the stability of a measure across different sets of items (Anastasi, 1988). In the split-half method, stability is assessed by dividing a single test into two halves (such as the oddand even-numbered items) and measuring the association between the scores obtained from each half. Another method is to assess alternate-forms stability, which refers to the association between two scores obtained from two forms of a test that are similar but contain different items (the two tests are administered at different test sessions). Note that both split-half and alternate-forms stability are based on a comparison between two measures obtained from different sets of items.

Although an individual's metamemory judgments exhibit excellent reliability when a given set of items is presented twice, there are few data on the stability of measures that assess the degree to which metamemory judgments predict memory performance (i.e., the stability of metamemory accuracy). Some previous research suggests that measures of metamemory accuracy may not be stable when different items are used on each test. In two studies of memory for general knowledge, Nelson (1988) found no stability in FOK accuracy. For example, in one condition subjects attempted to answer questions until they failed to answer 110 questions. FOK ratings were obtained for these questions, and a 7-alternative forced-choice recognition test was then administered. To measure FOK accuracy, a separate correlation between FOK ratings and recognition accuracy was computed for the odd- and even-numbered items on the test. This allowed an estimate of split-half stability to be computed by correlating FOK accuracy obtained from the odd and even items, and this stability coefficient was -.02 . Nelson obtained similarly low split-half estimates of stability for tests composed of 30 and 70 questions.

In the present research, our goal was to determine if people exhibit a stable relationship between memory performance and confidence judgments across different sets of test items. In Experiments 1 and 2 we assessed the stability of this relationship for recognition memory with three types of material: photographs of faces, words, and general knowledge questions. In Experiment 3 we examined the split-half stability of the association between confidence judgments and the accuracy of recalled answers to general knowledge questions.

\section{EXPERIMENT 1}

Experiment 1 was designed to assess the split-half and alternate-forms stability of the association between confidence judgments and recognition performance. At two experimental sessions separated by a 2-week interval, subjects studied photographs of faces and words, and then took old-new recognition tests over these items. The students also rated how confident they were in each answer they provided on the recognition tests. In addition, the students answered true-false general knowledge questions at both sessions, and rated how confident they were in each answer. Different sets of faces, words, and questions were used at each test session.

The Goodman-Kruskal gamma coefficient was used to assess the degree of association between confidence judgments and recognition performance (see Nelson, 1984). Gamma correlations were computed for each subject between the confidence judgments and the accuracy of each response on the recognition test (we refer to this as the confidence-recognition, or $\mathrm{CR}$, association). To assess the stability of the CR association for different items within a test (i.e., split-half stability) we examined the Spearman $\rho$ correlation between $\mathrm{CR}$ gammas computed separately for the odd- and even-numbered test items. The degree of splithalf stability in the CR association would be reflected in the degree to which there is a monotonic relationship between the individuals' $C R$ gammas for the odd-numbered items and their CR gammas for the even-numbered items. CR gammas were also computed at both sessions, using all items on each recognition test. An examination of the association between these pairs of CR gammas allowed us to assess the alternate-forms stability of the CR associa- 
tion, that is, stability across two different sets of items administered 2 weeks apart.

\section{Method}

Subjects. The subjects were 44 students from lower level psychology courses who received extra credit points for their participation.

Materials. The general knowledge test was made up of 80 questions adapted from Nelson and Narens (1980). The questions were written in true-false format and varied from easy (e.g., "The name of Tarzan's girl friend was Jane.") to difficult (e.g., "Gallant Fox was racehorse of the year for many successive years in the 1960s."). Because half the statements were true, students had a $50 \%$ chance of being correct even when guessing.

For the face recognition task, 80 photographs of white males were taken from a college yearbook. Photos containing glasses or facial hair were not used. This relatively homogeneous set of faces was used to create a difficult recognition test in order to avoid a ceiling effect on recognition performance.

For the word recognition task, 80 trait adjectives were taken from the high meaningfulness subset of the Anderson (1968) norms. As with the faces, these words were used to avoid a performance ceiling. Forty of the words had likability ratings above 4.00 (e.g., friendly, warm), and 40 had likability ratings below 2.00 (e.g., greedy, rude).

Faces were used as stimuli to provide a comparison with previous face memory and eyewitness research, and the word recognition test allowed a comparison with previous verbal learning studies. The data from the general information questions were sought to allow a comparison with other studies in which they have been used to examine the association between confidence judgments and memory performance (e.g., Costermans et al., 1992; Kelley \& Lindsay, 1993).

Two sets of test materials (A and B) were assembled randomly, each containing 40 true-false questions ( 20 true), 40 faces ( 20 targets), and 40 words ( 20 targets). The students were tested with one set, then were retested 2 weeks later with the other set. Out of the 44 students who were tested twice, 20 saw Set A first, then Set B, and the remaining 24 students were first tested with Set $B$, then retested with Set A.

Procedure. First, subjects studied the 20 faces. Slides of the faces were shown individually at a 5 -sec rate. Next, 20 trait adjectives were shown individually on slides at a 5-sec rate. For both the faces and words, we informed the subjects that there would be a recognition test later in the session. The general information test was given next. The questions were read aloud by the experimenter, one every $10 \mathrm{sec}$. For each question, subjects circled "true" or "false" on their answer sheet and rated how confident they were in their answer on a scale from 0 (guessing) to 3 (certain). The subjects were told that half the items were true and half were false.

Next, the subjects were given an old-new recognition test for the faces. The 20 target faces were randomly mixed with the 20 distractor faces and displayed one at a time at a 10 -sec rate. For each face, subjects indicated "old" or "new" and rated their confidence in the answer on a scale from 0 to 3 . Finally, the subjects were tested for their recognition of the words. The 20 target words were randomly mixed with the 20 distractor words and presented at a $10-\mathrm{sec}$ rate. The subjects marked "old" or "new" for each word and rated their confidence on the same 4-point scale used in the other two tasks. For both tests, the subjects knew that half the items were old and half were new.

Two weeks later the subjects returned and the entire procedure was repeated with the appropriate new set of materials (A or B).

\section{Results and Discussion}

To measure the CR association, we computed GoodmanKruskal gamma coefficients $(G)$ for each subject using the 40 pairs of confidence ratings and response correctness on each memory task. There was a large range in the degree of association between confidence and recognition per-
Table 1

Means and Standard Deviations for Confidence-Recognition (CR) Gamma, Proportion Correct, and Median Confidence Rating on the Three Memory Tasks in Experiment 1

\begin{tabular}{|c|c|c|c|c|c|c|}
\hline & \multicolumn{2}{|c|}{ Questions } & \multicolumn{2}{|c|}{ Faces } & \multicolumn{2}{|c|}{ Words } \\
\hline & $M$ & $S D$ & $M$ & $S D$ & $M$ & $S D$ \\
\hline \multicolumn{7}{|c|}{ Test I Memory Task } \\
\hline CR gamma & .48 & .47 & .36 & .45 & .49 & .25 \\
\hline Proportion correct & .67 & .11 & .78 & .13 & .78 & .09 \\
\hline Median confidence* & 2.11 & .85 & 2.47 & .84 & 2.46 & .69 \\
\hline \multicolumn{7}{|c|}{ Test 2 Memory Task } \\
\hline CR gamma & .49 & .35 & .40 & .38 & .50 & .25 \\
\hline Proportion correct & .69 & .10 & .81 & .11 & .75 & .11 \\
\hline Median confidence* & 2.39 & .84 & 2.43 & .72 & 2.14 & .81 \\
\hline
\end{tabular}

*Confidence ratings were made on a scale from 0 to 3 .

formance. On Test 1, $G$ for the questions ranged from -.47 to .92 , although only 2 subjects had negative $G$ s. $G$ ranged from -1.00 to 1.00 ( 4 were less than 0 ) on the face recognition task, and from -1.00 to .93 on the word recognition task, with seven negative $G$ s. Similar ranges occurred in the $G$ s obtained at the second testing session.

Table 1 gives descriptive statistics for $G$, recognition accuracy (proportion correct), and confidence ratings. Splithalf stability of the CR association was measured by finding $G$ separately for the odd and even items of each test, then computing the Spearman $\rho$ correlation between these two $G$ s. The first two lines of Table 4 show the 6 split-half stability correlations for recognition accuracy; the mean of these 6 correlations is .47 . The top two lines in Table 5 show the six split-half stability correlations for median confidence ratings; these correlations have a mean of .69.1 The CR association displayed the lowest split-half correlations, with a mean of .19 across the tasks and test sessions (see the top two lines of Table 6).

To measure alternate-form stability, we computed Spearman $\rho$ correlations between the students' performance on the first and second administrations of the 3 memory tasks. The alternate-forms correlations for recognition accuracy, median confidence rating, and CR gamma are shown in the next-to-last line of Tables 4,5 , and 6 , respectively. The mean of the 3 stability correlations for CR association was 0 , indicating a lack of stability in the CR association across test sessions. This result is consistent with previous research that also found no stability in the accuracy of metacognitive judgments across different sets of items (Glenberg \& Epstein, 1987; Nelson, 1988).

Examination of the scatterplots of the split-half and alternate-forms correlations for $G$ shows that the lack of stability is not due to a restricted range in either $G$ or a nonmonotonic relationship between the $G$ s (Figures 1-2).

\section{EXPERIMENT 2}

In Experiment 1 we found a lack of stability in the association between confidence judgments and recognition performance, and this instability occurred both between two halves of the same tests and between two alternate forms 

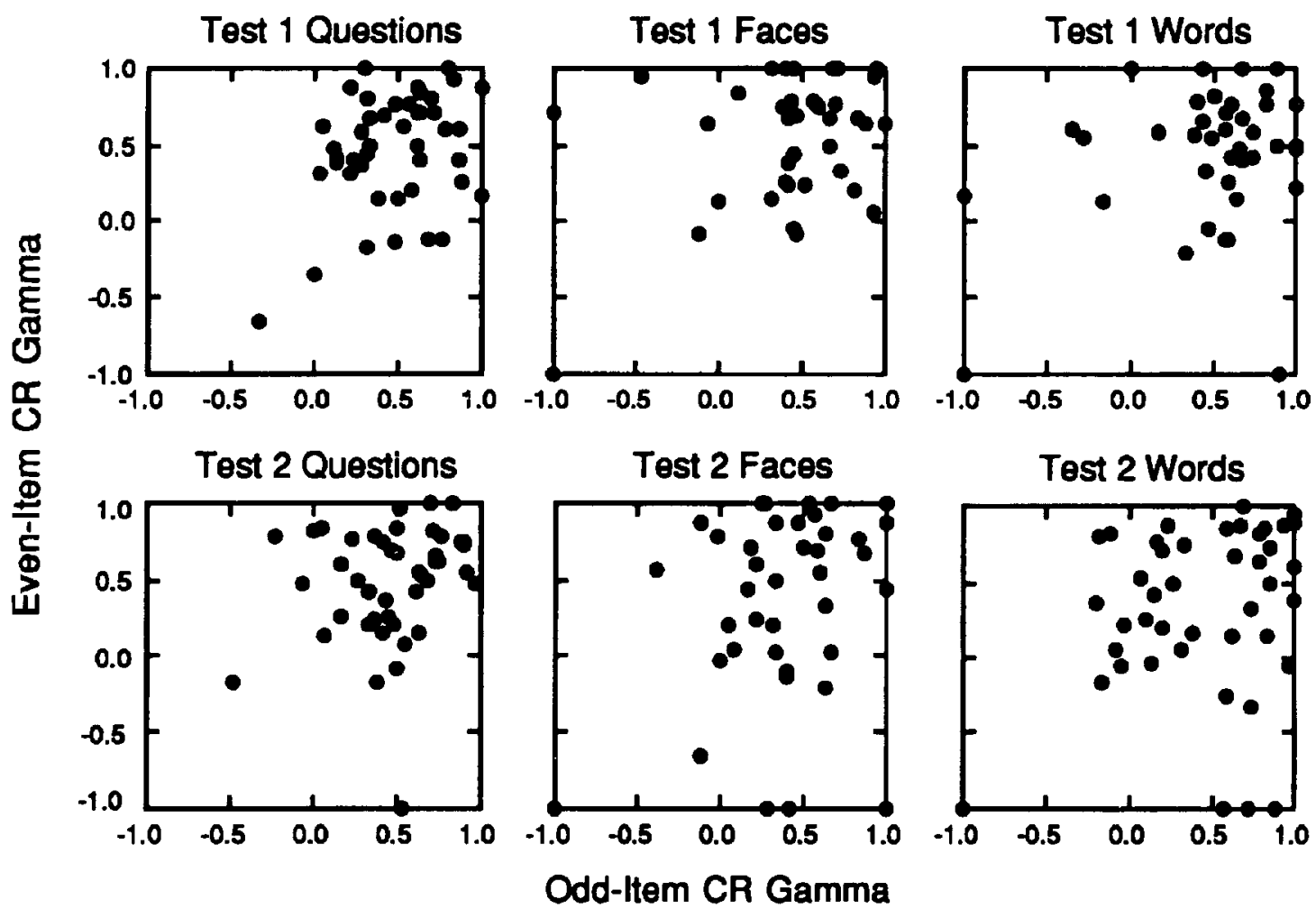

Figure 1. Scatterplots showing the relationship between individuals' confidence-recognition (CR) gammas based on odd- and even-numbered test items in Experiment 1. Each point on the scatterplots shows an individual subject's CR gamma for the odd-numbered items plotted against that individual's CR gamma for the even-numbered items.

of the tests. Experiment 2 was designed to see if the results of Experiment 1 could be extended to a population other than college students. We chose to test elderly adults not only because they represent a different population, but also because they generally show more variation in cognitive task performance than college students (Kausler, 1982; Krauss, 1980). Although we observed much variability in the accuracy, confidence, and CR association in students, it is possible that even more variance would be present in elderly adults. Thus, our intent was to see if our failure to observe stability in the CR association was in part caused by too little variation in recognition accuracy or confidence ratings in our sample of college students. Also, the procedure in the first experiment did not counterbalance the order in which the memory tests for general knowledge, faces, and words were given, so this was corrected in Experiment 2.

\section{Method}

Subjects. The subjects were 34 adults ( 8 males) between the ages of 55 and $85(M=67.4)$. Four were recruited by contacting alumni, and the rest were members of a senior citizens' center. Each subject was paid $\$ 10$ upon completion of the study.

Materials and Procedure. Except for the differences noted below, the materials and procedures were the same as those in Experiment 1 . Subjects were tested individually or in small groups. At each test session the subjects first studied the 20 target faces and 20 target words. Half the subjects studied the words first, and half studied the faces first. The faces and words were presented in booklets with 4 items per page and subjects were given $30 \mathrm{sec}$ to study each page. ${ }^{2}$ After a 10 -min retention interval, during which a questionnaire on everyday memory experiences was completed, the subjects were tested on their memory for faces, words, and general knowledge. On the 40 -item true-false general knowledge test, subjects marked "true" or "false" on their answer sheet and rated how confident they were in their answer on a scale from 0 (guessing) to 4 (certain). We used this 5-point scale, rather than the 4-point scale from Experiment 1, because we thought it would allow for more variability in confidence judgments. On the recognition tests for faces and words, the 20 target items were randomly mixed with 20 distractor items and were presented in booklets with 10 items per page. For each item, the subjects indicated "old" or "new," then rated their confidence in the answer on a scale from 0 to 4 . The order of test administration (faces, words, and questions) was counterbalanced across subjects by randomly assigning each individual or group to one of the six possible test sequences.

\section{Results and Discussion}

Table 2 gives summary statistics for the $G$ s between confidence ratings and recognition accuracy, recognition accuracy (proportion correct), and median confidence ratings; scores among individuals varied approximately as much as those in Experiment 1 (compare the standard deviations in Tables 1 and 2). The split-half stability correlations for proportion correct at each testing session are shown in the third and fourth lines of Table 4 . The split-half correlations for median confidence can be found in Table 5 . Whereas proportion correct and median confidence showed moderate to good stability, the stability of the CR association was low, 


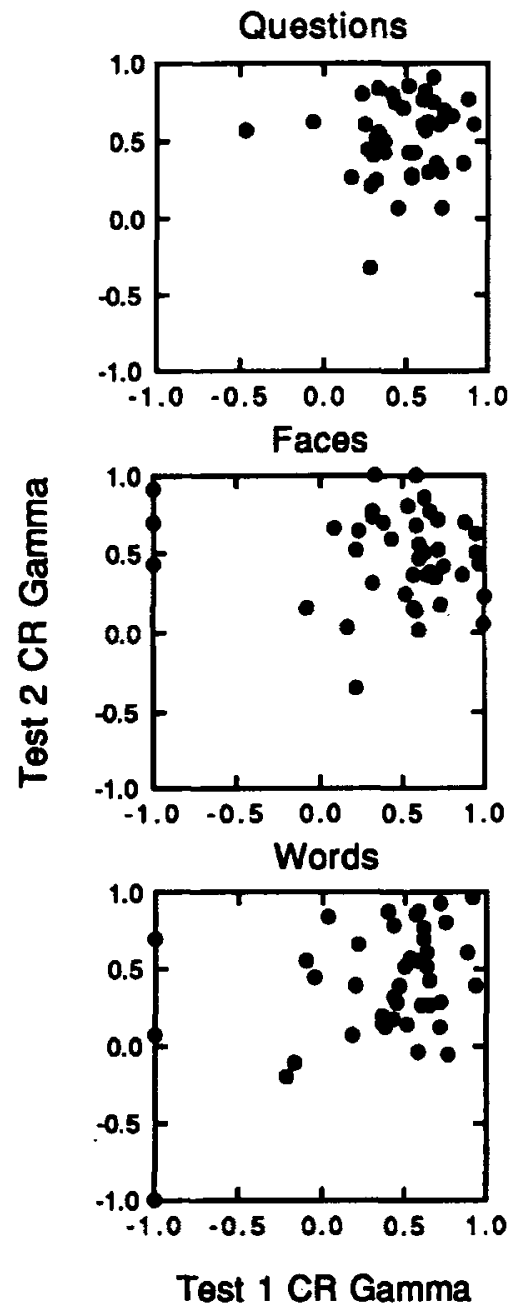

Figure 2. Scatterplots of the relationship between individuals' confidence-recognition (CR) gammas from the first and second test sessions in Experiment 1, for each item type (questions, faces, and words). Each point shows an individual subject's CR gamma based on items presented at the first test session plotted against that individual's corresponding CR gamma from the second test session.

even compared to the modest values found in Experiment 1; only the split-half correlation for questions at the second testing session was significantly different from 0 (Table 6).

The association between confidence judgments and recognition performance showed no stability from the first to the second test session; none of the alternate-forms correlations was significantly different from 0 (see the last line of Table 6). These results generally replicate the findings of Experiment 1 and suggest that the lack of stability in individuals' $\mathrm{CR}$ association observed in Experiment 1 was not due to the homogeneity of the student sample.

\section{EXPERIMENT 3}

Experiments 1 and 2 indicated that the association between confidence and recognition performance was not stable across different sets of items (either within the same test or between tests given at different times). Because both studies involved recognition tests of memory, we designed Experiment 3 to see if similar results would occur if a recall test was used. It is possible that the old-new and true-false recognition tests we employed in Experiments 1 and 2 contributed to the low stability we observed because the probability of correctly answering a question was . 50 even in the absence of any knowledge about an item. To reduce this source of error variance in memory performance, we had subjects recall answers to general knowledge questions in Experiment 3.

\section{Method}

Subjects. The subjects were 70 volunteers from undergraduate psychology classes who received bonus points in return for their participation. The students were tested in groups of 3 to 9 .

Materials and Procedure. The subjects answered the same 80 general knowledge questions as those used in Experiments 1 and 2, except the questions were open-ended rather than true-false. The questions were presented in random order in a booklet, with 25 questions on each of the first 3 pages and 5 questions on the last page, and the subjects wrote their answers and confidence ratings in blanks on a separate answer sheet. The students were instructed to write down an answer only if they thought there was at least a small chance the answer was correct. The students rated confidence in each answer on a 4-point scale $(1=$ not at all sure my answer is correct $; 4=$ very sure my answer is correct).

\section{Results and Discussion}

Test performance was analyzed for the entire 80question test and separately for the odd-and even-numbered questions (see Table 3 for a summary). The students answered an average of 46.5 of the 80 questions, and an average of $56 \%$ of these answers were correct. Question difficulty was very evenly distributed: 26 questions were answered correctly by no more than one third of the students; another 30 questions were answered correctly by between one third and two thirds of the students; 24 questions were answered correctly by at least two thirds of the students.

As in the first two experiments, the main dependent measure was the degree of association between confidence ratings and memory performance, in this case the correctness of each recalled answer. For each subject, we computed one overall confidence-recall $G$ based on all questions for which the subject recalled an answer. These overall $G$ s ranged

Table 2

Means and Standard Deviations for Confidence-Recognition (CR) Gamma, Proportion Correct, and Median Confidence Rating on the Three Memory Tasks in Experiment 2

\begin{tabular}{|c|c|c|c|c|c|c|}
\hline & \multicolumn{2}{|c|}{ Questions } & \multicolumn{2}{|c|}{ Faces } & \multicolumn{2}{|c|}{ Words } \\
\hline & $M$ & $S D$ & $M$ & $S D$ & $M$ & $S D$ \\
\hline \multicolumn{7}{|c|}{ Test 1 Memory Task } \\
\hline CR gamma & .44 & .28 & .22 & .34 & .36 & .40 \\
\hline Proportion correct & .73 & .11 & .69 & .10 & .71 & .13 \\
\hline Median confidence* & 2.72 & 1.14 & 2.79 & 1.10 & 3.12 & .76 \\
\hline \multicolumn{7}{|c|}{ Test 2 Memory Task } \\
\hline CR gamma & .50 & .24 & .22 & .26 & .14 & .50 \\
\hline Proportion correct & .73 & .11 & .67 & .11 & .72 & .14 \\
\hline Median confidence* & 2.69 & 1.05 & 2.82 & .96 & 3.00 & .78 \\
\hline
\end{tabular}

*Confidence ratings were made on a scale from 0 to 4 . 
Table 3

\begin{tabular}{|c|c|c|c|c|c|c|}
\hline & \multicolumn{2}{|c|}{ All Questions } & \multicolumn{2}{|c|}{ Odd Questions } & \multicolumn{2}{|c|}{ Even Questions } \\
\hline & $M$ & $S D$ & $M$ & $S D$ & $M$ & $S D$ \\
\hline No. of answers & 46.5 & 11.0 & 24.2 & 5.84 & 22.3 & 5.88 \\
\hline Confidence-recall gamma & .75 & .13 & .77 & .17 & .75 & .19 \\
\hline Proportion correct & .56 & .14 & .58 & .14 & .53 & .17 \\
\hline Median confidence* & 3.03 & .82 & 3.17 & .84 & 2.91 & .84 \\
\hline
\end{tabular}

from .41 to .95 . We also computed one confidence-recall $G$ using only the odd-numbered questions and another $G$ based on the even-numbered questions (using the item numbers from the original test). These data are summarized in Table 3.

Estimates of split-half stability for the 80-question test were obtained by calculating the Spearman $\rho$ correlation between subjects' confidence-recall $G$ s from the odd and even halves of the tests. In general, the pattern of results was consistent with that of Experiments 1 and 2. The Spearman correlation between the median confidence ratings on the odd- and even-numbered items was .79 ( $p<$ $.05)$, and the Spearman correlation was $.56(p<.05)$ between recall accuracy scores (proportion correct) on the odd- and even-numbered halves of the test. In contrast to these strong correlations, the split-half correlation for confidence-recall association was only .04. An examination of the scatterplot for the odd and even $G$ s showed that this lack of stability was not caused by a restricted range on the $G$ s or by a nonmonotonic relationship between the two measures (Figure 3).

\section{GENERAL DISCUSSION}

The purpose of this research was to assess the degree of stability in the association between confidence judgments and performance on recall and recognition tasks. Our major finding was that this association, as measured by the Goodman-Kruskal gamma coefficient, showed almost no stability across different sets of items. Table 6 summarizes the alternate-forms and split-half stability correlations from Experiments 1-3. Across the experiments, the mean of the 13 split-half stability coefficients was only .15 . With such low split-half stability, it is not surprising that the mean of the 6 alternate-forms stability estimates from Experiments 1 and 2 was also very low, at .02. Our use of a recall test in Experiment 3, to reduce error variance in memory performance due to guessing, did not increase the stability of the association between confidence and memory performance. These data are consistent with previous research by Nelson (1988), who found no stability for FOK accuracy, and research by Glenberg and Epstein (1987), who reported a lack of stability in a measure of metacognitive accuracy (as described in the next paragraph) across two sets of items.

These findings suggest that future studies should be designed to clarify which characteristics of specific test items affect metamemory accuracy. The present data also indicate that current techniques appear to be inadequate for the assessment of changes in individual metamemory accuracy when the same test items cannot be used twice. This lack of metamemory stability presents a problem for researchers attempting to discover the origins of individual differences in metacognitive accuracy. As an example, consider the study by Glenberg and Epstein (1987), in which subjects read 32 paragraphs, 16 on topics in music and 16 on topics in physics. Later, the subjects decided if inferences based on the readings were correct or incorrect and rated how confident they were in their decisions. Glenberg and Epstein measured the degree of association between the confidence ratings and performance on the inference test. They found that this measure of metacognitive accuracy was unrelated to an individual's degree of expertise in physics or music. Glenberg and Epstein also found no relationship between a different measure of metacognitive accuracy and a measure of individual differences in epistemological style.

Similarly, Maki and Swett (1987) found no relationship between two measures of metamemory accuracy. These researchers had college students read stories and rate how likely they were to recall the main ideas in the stories. Later, the students tried to recall the ideas from the stories and also rated how confident they were that each idea had

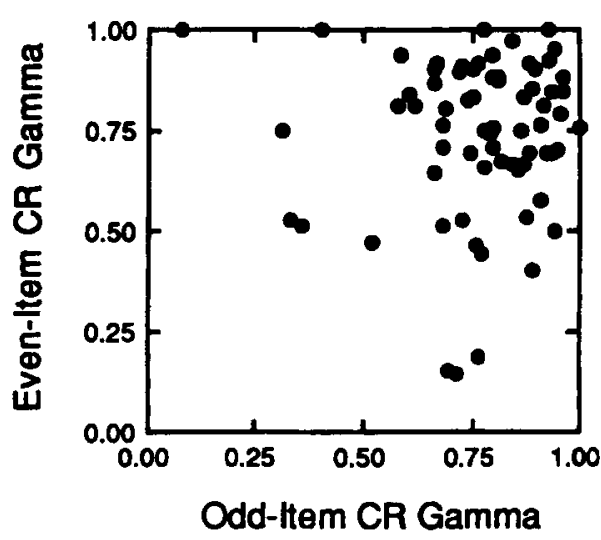

Figure 3. Scatterplot showing the relationship between individuals' confidence-recall gammas based on odd- and even-numbered questions in Experiment 3. Each point on the scatterplot shows an individual subject's confidence-recall gamma for odd-numbered questions plotted against that individual's confidence-recall gamma for even-numbered questions. 
Table 4

Summary of Split-Half and Alternate-Forms Correlations (Spearman $\rho$ ) for Proportion Correct From Experiments 1-3

\begin{tabular}{lccc}
\hline & \multicolumn{3}{c}{ Memory Task } \\
\cline { 3 - 4 } & Questions & Faces & Words \\
\hline Split-Half Stability & & & \\
Experiment 1, Test 1 & $.34^{*}$ & $.60^{*}$ & $.36^{*}$ \\
Experiment 1, Test 2 & $.36^{*}$ & $.56^{*}$ & $.57^{*}$ \\
Experiment 2, Test 1 & $.59^{*}$ & $.41^{*}$ & $.58^{*}$ \\
Experiment 2, Test 2 & $.49^{*}$ & $.47^{*}$ & $.63^{*}$ \\
Experiment 3 (Recall) $\dagger$ & $.56^{*}$ & & \\
Alternate-Forms Stability & & & \\
Experiment 1 & $.48^{*}$ & $.57^{*}$ & .18 \\
Experiment 2 & $.62^{*}$ & .13 & $.52^{*}$ \\
\hline
\end{tabular}

*Significantly different from 0 at the .05 alpha level. ${ }^{\dagger}$ A single recall test was administered in Experiment 3; recognition tests were used in Experiments 1-2.

Table 5

Summary of Split-Half and Alternate-Forms Correlations (Spearman $\rho$ ) for Median Confidence Rating From Experiments 1-3

\begin{tabular}{lccc}
\hline & \multicolumn{3}{c}{ Memory Task } \\
\cline { 2 - 4 } & Questions & Faces & Words \\
\hline Split-Half Stability & & & \\
Experiment 1, Test 1 & $.65^{*}$ & $.73^{*}$ & $.80^{*}$ \\
Experiment 1, Test 2 & $.64^{*}$ & $.67^{*}$ & $.65^{*}$ \\
Experiment 2, Test 1 & $.75^{*}$ & $.95^{*}$ & $.85^{*}$ \\
Experiment 2, Test 2 & $.44^{*}$ & $.85^{*}$ & $.67^{*}$ \\
Experiment 3 (Recall) $\dagger$ & $.79^{*}$ & & \\
Alternate-Forms Stability & & & \\
Experiment 1 & $.55^{*}$ & $.55^{*}$ & $.74^{*}$ \\
Experiment 2 & .24 & $.48^{*}$ & $.56^{*}$ \\
\hline
\end{tabular}

${ }^{*}$ Significantly different from 0 at the .05 alpha level. ${ }^{\dagger} A$ single recall test was administered in Experiment 3; recognition tests were used in Experiments 1-2.

actually appeared in the stories. For each subject, Maki and Swett computed one measure of metacognitive accuracy (prediction accuracy) that assessed the degree of association between recall performance and the recall prediction ratings. A second measure of metacognitive accuracy assessed the degree of association between recall performance and postrecall confidence ratings. There was no correlation between these two measures of metacognitive accuracy. In each case, the failure to find a relationship between metacognitive accuracy and another factor might have been partly due to a lack of stability in the estimates of individuals' metacognitive accuracy.

We also found that confidence ratings exhibited greater stability than actual recognition or recall performance. This suggests that the low correlations between confidence and memory performance obtained in many studies of face recognition and eyewitness memory may be due in part to the stability of subjects' confidence ratings relative to their accuracy. Confidence ratings do not vary solely as a function of those processes that determine the accuracy of a memory, and this reduces the degree of association between confidence ratings and memory performance.
Assuming people have stable metacognitive accuracy, why did we fail to observe that stability in the present experiments? One possibility is that our tests contained too few items. In Experiments 1 and 2, each subject's CR gammas were based on either 20 or 40 items. In Experiment 3 , confidence-recall gammas for the odd- and even-numbered items were based on an average of 24 and 22 items, respectively. One indication that we may have used too few items was the occurrence of negative $C R$ gammas for some subjects (although the majority of subjects had CR gammas between 0 and 1 on both halves of the tests and on the two forms of the tests; this can be seen in Figures 1-2, in which most of the points are in the upper-right quadrant of the scatterplots).

A low number of test items might lead to low stability in metamemory accuracy by producing low reliability in memory performance, especially given our use of old-new and true-false recognition tests in Experiments 1 and 2 (for which the probability of a correct guess was .50). In the present studies, the split-half and alternate-forms reliability estimates were not high for recognition and recall performance. For example, in Experiment 1 the mean of the 6 split-half stability correlations was .47 , and the mean of the 3 alternate-forms reliabilities was .41 (Table 4). Perhaps tests with very high reliability for memory performance must be employed before we will observe stability in metamemory accuracy. As one reviewer pointed out, gamma (or any other measure of association) contains error from the measurement of memory performance and confidence judgments, and the stability of metamemory accuracy was assessed by correlating pairs of gammas, each of which contains error from these two sources. Thus, it may be that to maximize the stability of measures of metamemory accuracy, the measurement of memory performance and metamemory judgments must be highly reliable.

In addition to an insufficient number of test items, idiosyncratic differences in item difficulty may have contributed to the low reliability of memory performance. For example, in Experiments 1 and 2 we randomly divided 80

Table 6

Summary of Split-Half and Alternate-Forms Correlations (Spearman $\rho$ ) for Confidence-Recognition and Confidence Recall Gammas From Experiments 1-3

\begin{tabular}{lccc}
\hline & \multicolumn{3}{c}{ Memory Task } \\
\cline { 2 - 4 } & Questions & Faces & Words \\
\hline Split-Half Stability & .21 & .13 & .18 \\
Experiment 1, Test 1 & .16 & .21 & .27 \\
Experiment 1, Test 2 & .09 & .08 & .05 \\
Experiment 2, Test 1 & $.34^{*}$ & -.04 & .20 \\
Experiment 2, Test 2 & .04 & & \\
Experiment 3 (Recall) $\dagger$ & & & \\
Alternate-Forms Stability & .14 & -.13 & -.01 \\
Experiment 1 & -.09 & .08 & .14 \\
Experiment 2 &
\end{tabular}

*Significantly different from 0 at the .05 alpha level. ${ }^{\dagger}$ A single recall test was administered in Experiment 3; recognition tests were used in Experiments 1-2. 
photographs of faces into 2 sets of 40 (twenty of which were presented for study). It may be that discriminating old (studied) from new (unstudied) faces was difficult at both testing sessions for 1 subject, while another subject found the first set of faces easier to remember than the second set (perhaps because several faces resembled people known to that subject). Such idiosyncratic differences could reduce both alternate-forms and split-half reliability in memory performance and contribute to instability in estimates of metamemory accuracy across sets of items. Thus, it is possible that metamemory accuracy was accurately measured by gamma, and that the instability in the association between confidence judgments and memory performance across different sets of items was caused mainly by idiosyncratic differences in item difficulty (if so, other measures of association would also be unstable). This possibility suggests that stable measures of metamemory accuracy require sets of items and testing procedures that minimize idiosyncratic differences in item difficulty.

Although it seems plausible that stable estimates of metamemory accuracy require tests with high memory performance reliability, an examination of the reliabilities obtained in the present experiments reveals no clear relationship between metamemory stability and the reliability with which memory performance was measured. For example, in Experiment 2 (Table 6), split-half stability in metamemory accuracy was greatest for the questions on Test $2(\rho=.34)$, even though the reliability estimate for proportion correct for that test was lower than three others in Experiment 2. The tests over questions and words at Test 1 and the words at Test 2 display higher reliability for proportion correct and yet have a lower estimate of CR stability than the questions on Test 2. Thus, stable estimates of individual metacognitive accuracy may not be obtained simply by using a large number of items and minimizing the probability of correct guesses. Recall that Nelson (1988) found no split-half stability in FOK accuracy even when each gamma was based on as many as 55 items, and the chance of a correct guess was minimized with the use of a 7-alternative, forcedchoice recognition test. Indeed, Nelson (1988) observed no relationship between the number of items on a test (he used 30,70 , and 110 items) and the stability of FOK accuracy. Nelson's findings also suggest that increasing the reliability of memory performance does not necessarily result in more stable estimates of individual metamemory accuracy across different sets of items. Although we would expect the reliability of memory performance to increase with test length, Nelson found no increase in the stability of FOK accuracy as test length increased from 30 to 110 items.

It appears that the search for a procedure that will yield stable estimates of individual metamemory accuracy will not be easy. Although we may still discover that certain individuals exhibit stable differences in the degree of association between memory performance and metamemory judgments across different sets of items, additional research is needed to discover the test items and procedures that will reveal such stability. If a person displays a high level of metamemory accuracy on one test, what does this mean? Does it mean this person has consistently high metacognitive accuracy for this task? At this point it seems that all we can say is that we happened to find a set of items that produced a high level of metamemory accuracy for this individual; if we test this person again with a different set of items, the first test score provides little or no information about this person's metamemory accuracy on the second test. The present findings point to the need to develop tests of metamemory accuracy that display both split-half and alternate-forms stability. Until such tests are available, researchers investigating various factors that may affect, or correlate with, individual differences in metamemory accuracy should interpret with caution any failure to find an effect.

\section{REFERENCES}

Anastasi, A. (1988). Psychological testing. New York: Macmillan. ANDERSON, N. H. (1968). Likableness ratings of 555 personality-trait words. Journal of Personality \& Social Psychology, 9, 272-279.

BRIGHAM, J. C. (1990). Target person distinctiveness and attractiveness as moderator variables in the confidence-accuracy relationship in eyewitness identifications. Basic \& Applied Social Psychology, 11, 101-115.

Costermans, J., Lories, G., \& ANSAY, C. (1992). Confidence level and feeling of knowing in question answering: The weight of inferential processes. Journal of Experimental Psychology: Learning, Memory. \& Cognition, 18, 142-150.

DEFFENBACHER, K. A. (1980). Eyewitness accuracy and confidence: Can we infer anything about their relationship? Law \& Human Behavior, 4, 243-260.

DefFenbacher, K. A., LeU, J. R, \& Brown, E. L. (1981). Memory for faces: Testing method, encoding strategy, and confidence. American Journal of Psychology, 94, 13-26.

GLENBERG, A. M., \& EPSTEIN, W. (1987). Inexpert calibration of comprehension. Memory \& Cognition, 15, 84-93.

KAUSLER, D. H. (1982). Experimental psychology and human aging. New York: Wiley.

Kelley, C. M., \& LindSAY, D. S. (1993). Remembering mistaken for knowing: Ease of retrieval as a basis for confidence in answers to general knowledge questions. Journal of Memory \& Language, 32, 1-24.

Krauss, I. K. (1980). Between- and within-group comparisons in aging research. In L. W. Poon (Ed.), Aging in the 1980s (pp. 542-551). Washington, DC: American Psychological Association.

LEIPPE, M. R. (1980). Effects of integrative memorial and cognitive processes on the correspondence of eyewitness accuracy and confidence. Law \& Human Behavior, 4, 261-274.

Luus, C. A. E., \& Wells, G. L. (1994). Eyewitness identification confidence. In D. F. Ross, J. D. Read, \& M. P. Toglia (Eds.), Adult eyewitness testimony: Current trends and developments (pp. 348-361). New York: Cambridge University Press.

MAKI, R. H., \& SWETr, S. (1987). Metamemory for narrative text. Memory \& Cognition, 15, 72-83.

NELSON, T. O. (1984). A comparison of current measures of feeling-ofknowing accuracy. Psychological Bulletin, 95, 109-133.

Nelson, T. O. (1988). Predictive accuracy of the feeling of knowing across different criterion tasks and across different subject populations and individuals. In M. M. Gruenberg, P. E. Morris, \& R. N. Sykes (Eds.), Practical aspects of memory: Current research and issues (Vol. 1, pp. 190-196). New York: Wiley.

Nelson, T. O., Leonesio, R. J., Landwehr, R. S., \& Narens, L. (1986). A comparison of three predictors of an individual's memory performance: The individual's feeling of knowing versus the normative feeling of knowing versus base-rate item difficulty. Journal of Experimental Psychology: Learning, Memory, \& Cognition, 12, 279287.

Nelson, T. O., \& NARENS, L. (1980). Norms of 300 general-information questions: Accuracy of recall, latency of recall, and feeling-of-knowing ratings. Journal of Verbal Learning \& Verbal Behavior, 19, 338-368.

Perfect, T. J., Watson, E. L., \& Wagstaff, G. F. (1993). Accuracy of 
confidence ratings associated with general knowledge and eyewitness memory. Journal of Applied Psychology, 78, 144-147.

SEward, G. H. (1928). Reaction time as a measure of confidence. $A r-$ chives of Psychology, 99, 1-54.

Strong, E. K. (1912). The effect of length of series on recognition memory. Psychological Review, 19, 447-462.

STRONG, E. K. (1913). The effect of time interval on recognition memory. Psychological Review, 20, 339-372.

TRow, W. C. (1923). The psychology of confidence. Archives of Psychology, 67, 1-47.

Wells, G. L., \& Murray, D. M. (1984). Eyewitness confidence. In G. L. Wells \& E. F. Loftus (Eds.), Eyewitness testimony: Psychological perspectives (pp. 155-170). New York: Cambridge University Press.

\section{NOTES}

1. We also examined the reliability correlations for mean confidence ratings, and in all three experiments reported in this article, found the same general pattern of results that occurred with the median confidence ratings

2. Before running Experiment 2 we tested the new procedure (using booklets instead of photographic slides) on a sample of 82 college students and found that the pattern of results was very similar to that in Experiment 1.

(Manuscript received October 20, 1994; revision accepted for publication January 18, 1995.) 